دراسة إقتصادية لدوال الطلب على الواردات من المبيدات الزراعية

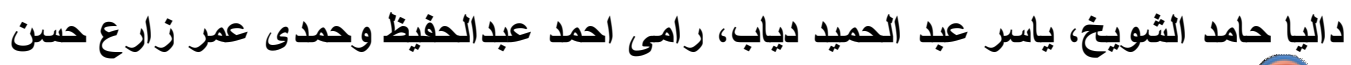

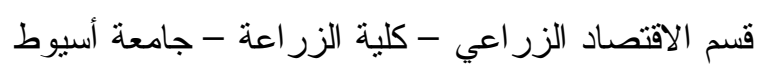

Received on: 1/9/2020

Accepted for publication on: 2/9/2020

مقدمة:

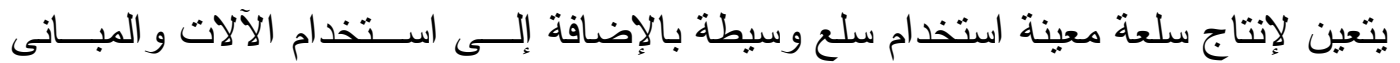

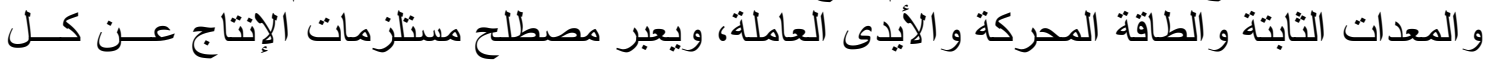

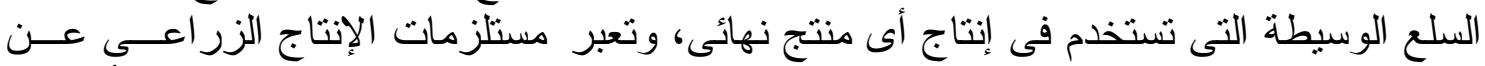

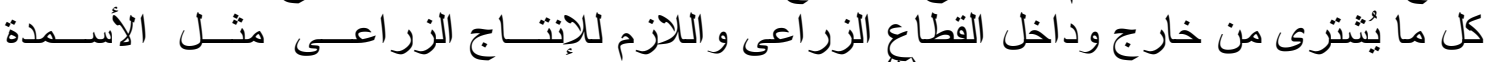

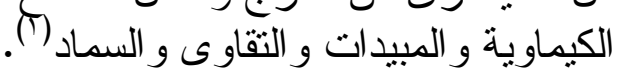

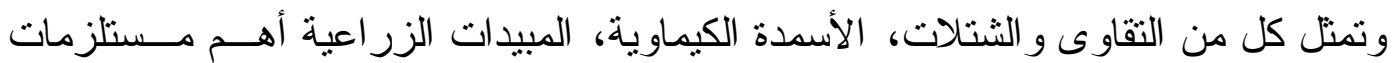

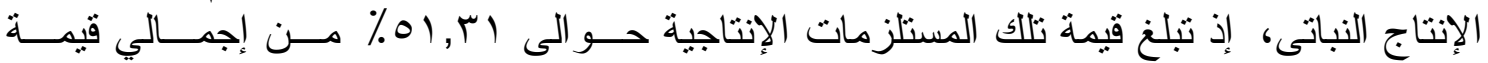

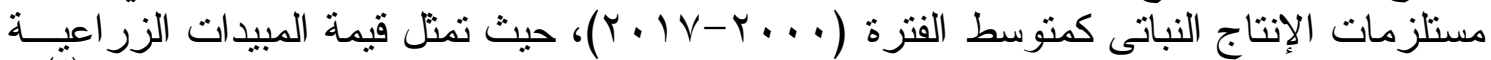

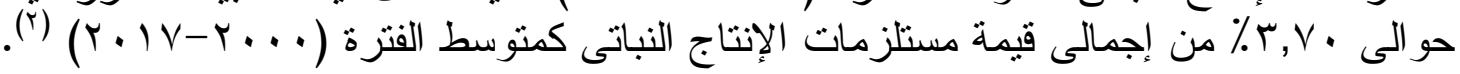

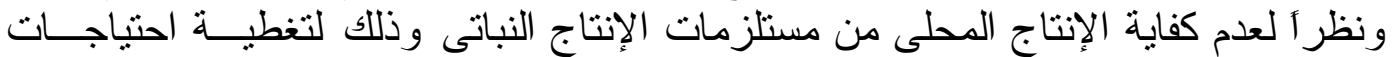

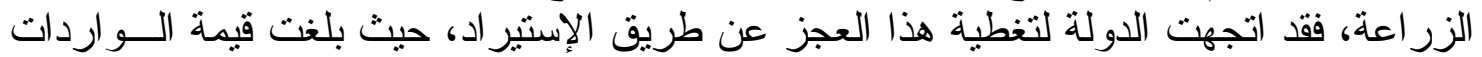

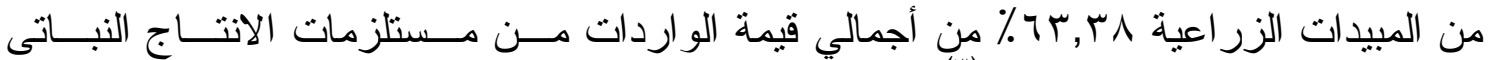

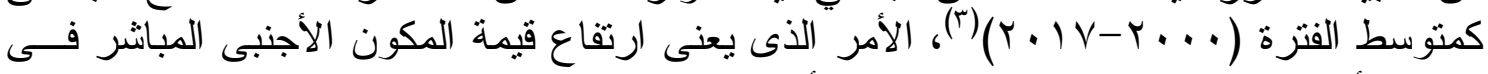

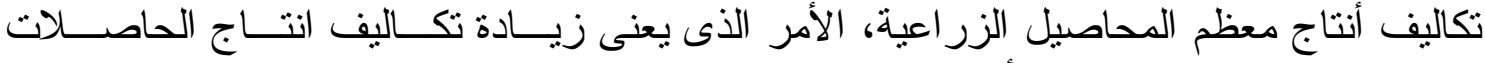
الزر اعية التى يشكل المكون الأجنبي المبانشر نسبة كبيرة من تكاليف الأنيف انتاجها.

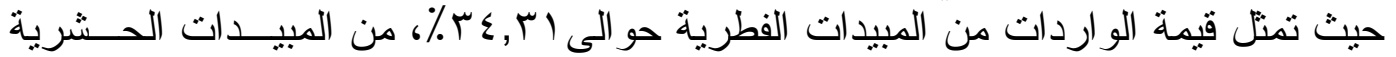

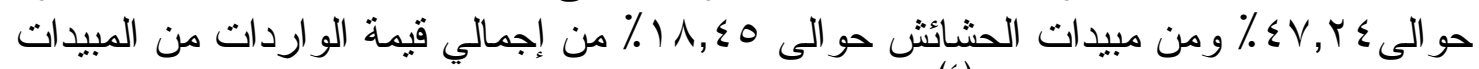

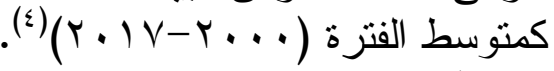
مشكلة البحث: - مثرة

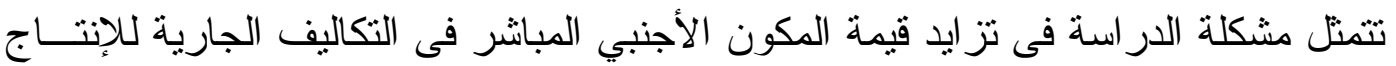

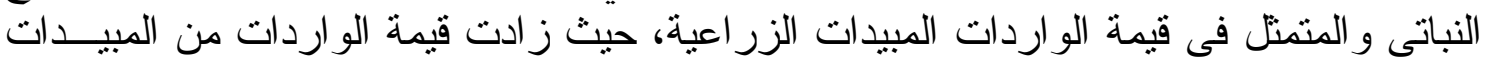

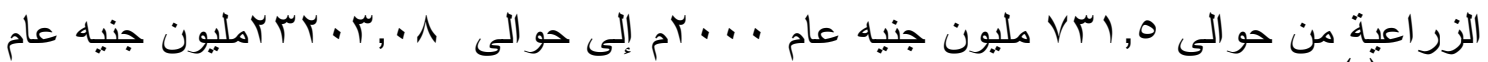

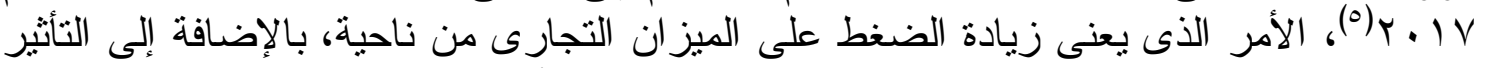

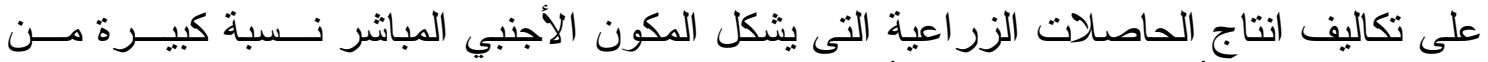

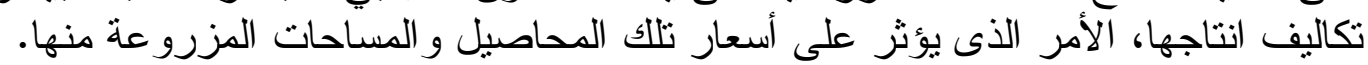

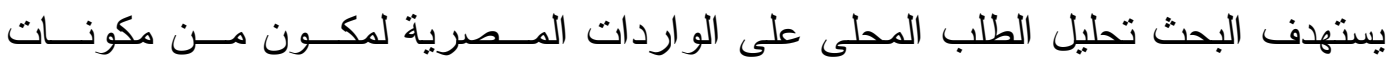

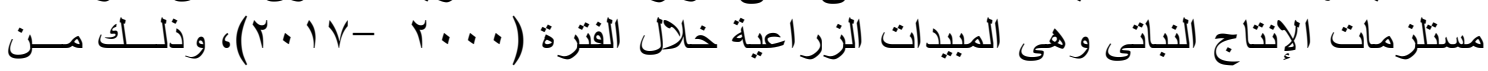

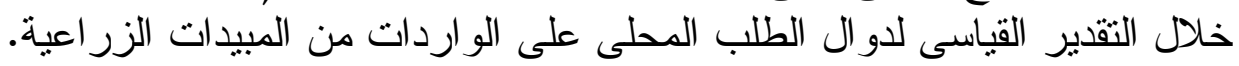

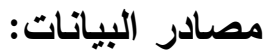

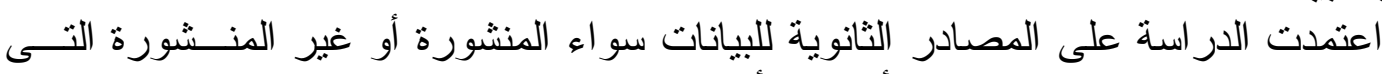

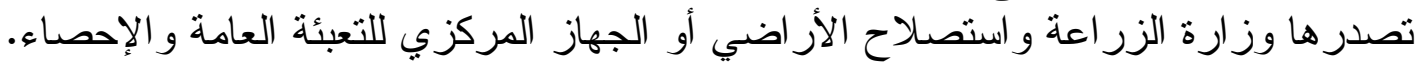

(') (؟) حسبت من الجهاز المركزى للتعبئة العامة والإحصاء، النشرة السنوية لتقدير ات الدخل من القطاع الزر اعى، أعداد متفرقة.

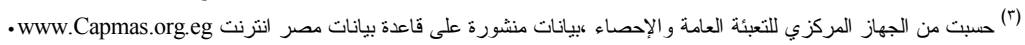

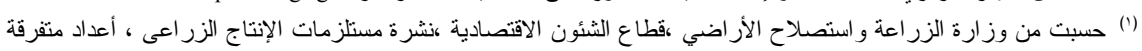

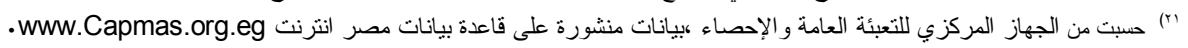




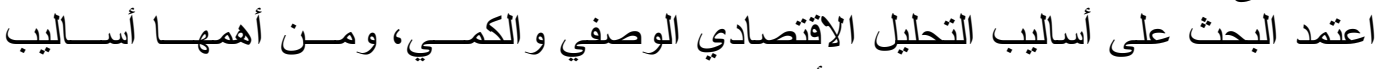

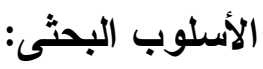

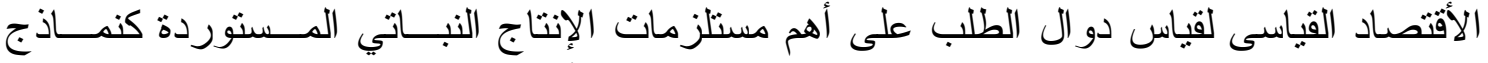

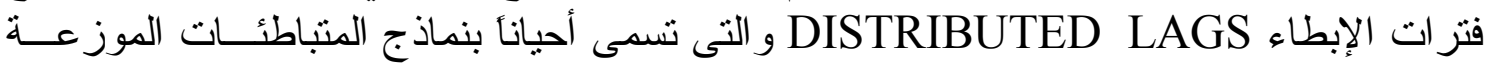

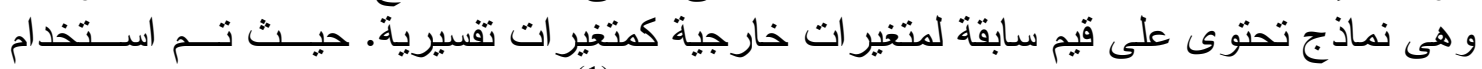

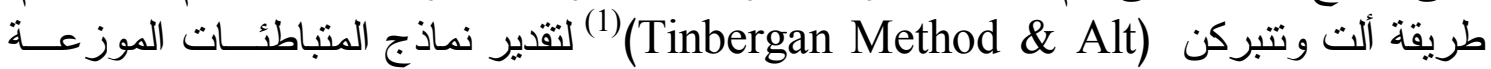

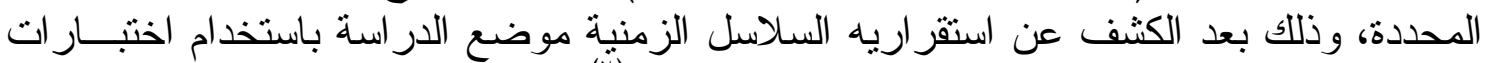

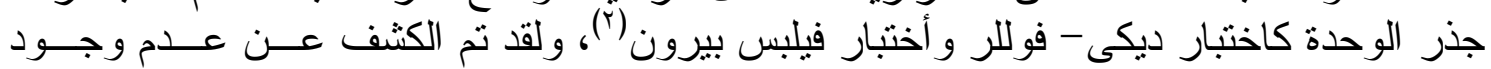
مشاكل قياسية بالنموذج التى قد يكون مصدر ها هو عدم التحقق من بعض الفرضيات الكلاسيكية

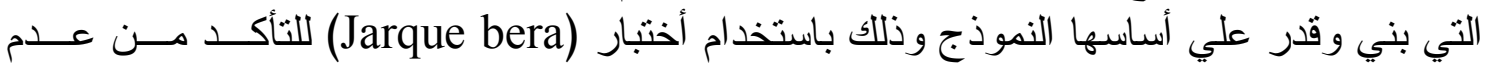

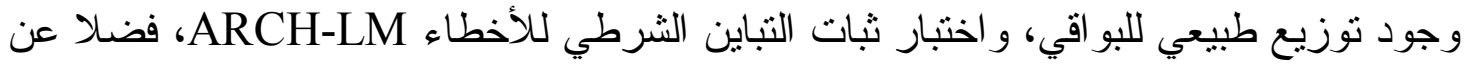

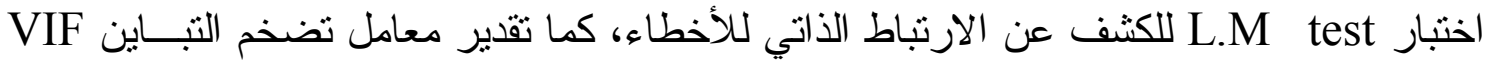
للكثف عن الارتباط المتعدد بين المتغير ات المستقلة. نتائج البحث: ألان:

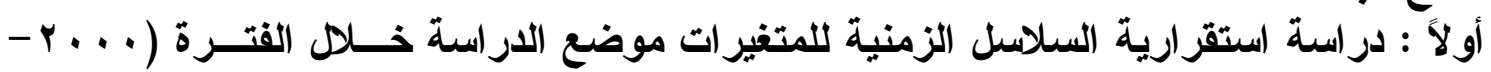

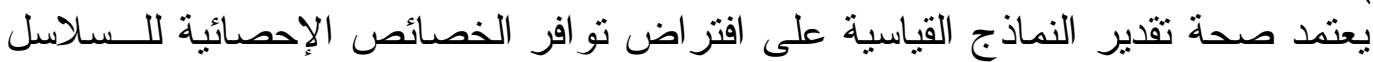

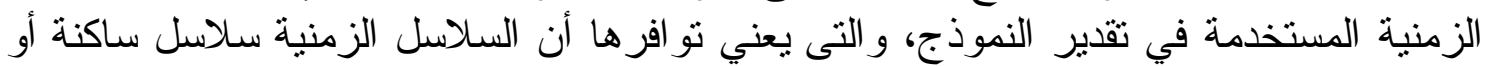

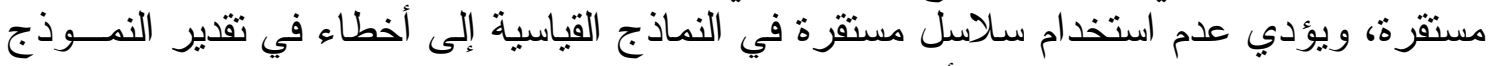

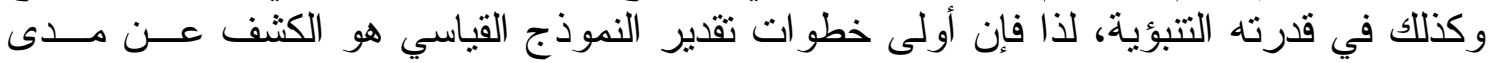

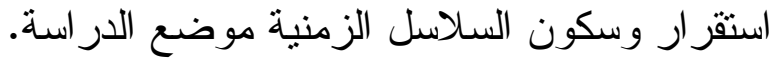

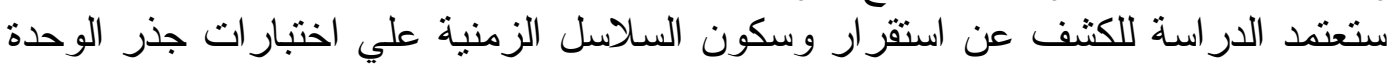

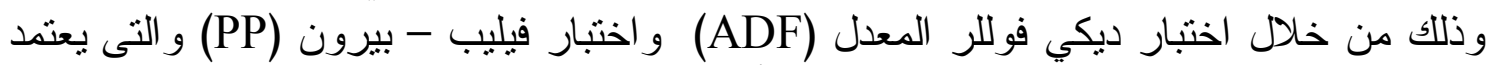
كل منهما على فرضية العدم والتى ينص على أن السلسلة الزمنية للمتغير غير ساكنة (يوجد فيها

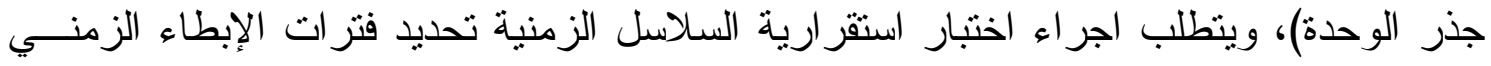

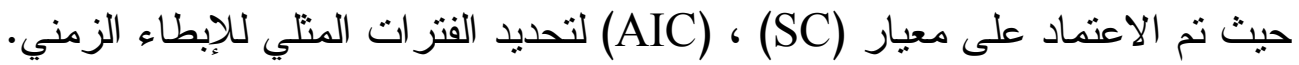

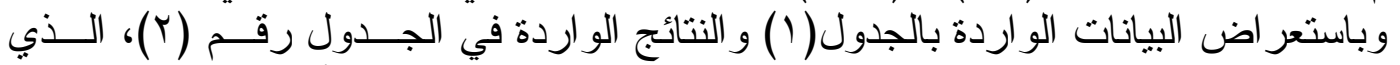

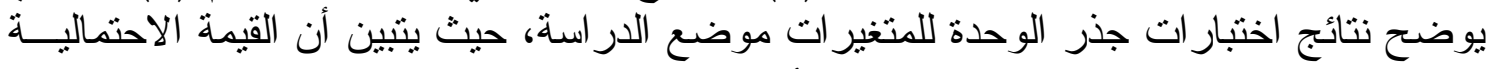

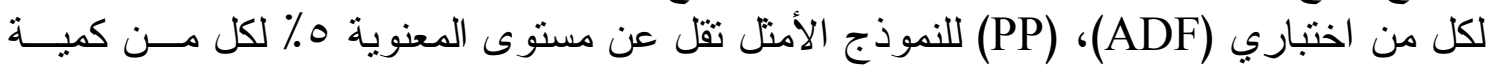

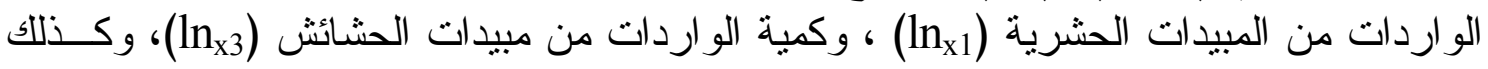

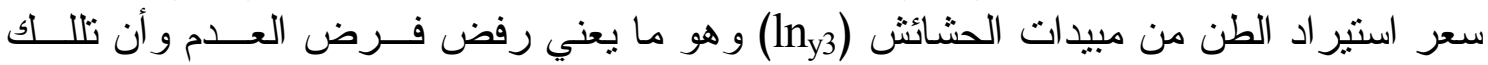

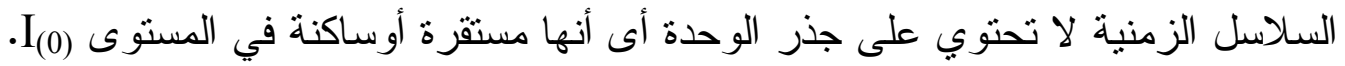

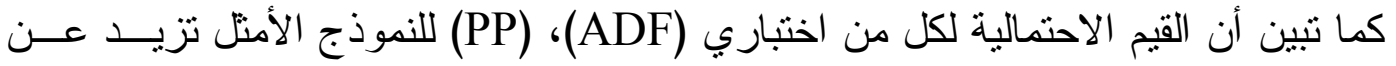
مستوى المعنوية ه\% لكل من كمية الو اردات من المبيدات الفطرية (X2) وسعر استير اد الطن من الطن

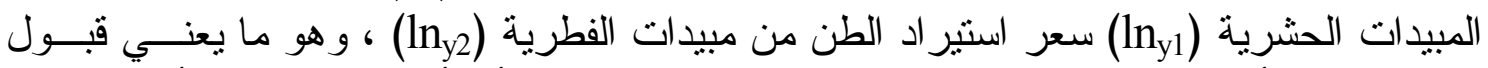

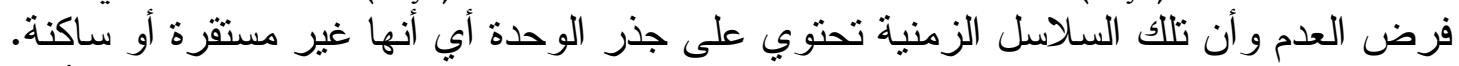

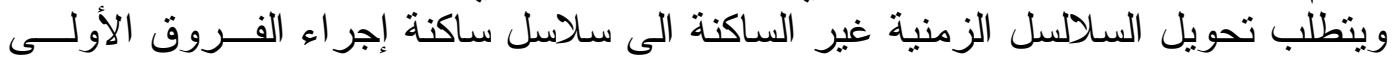

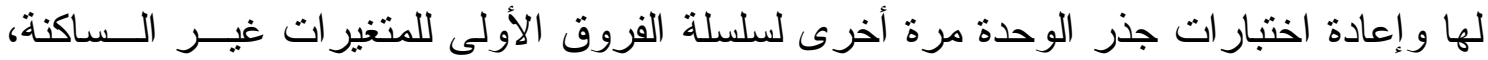




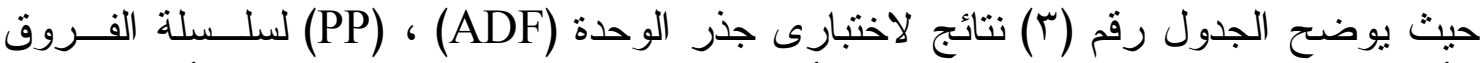

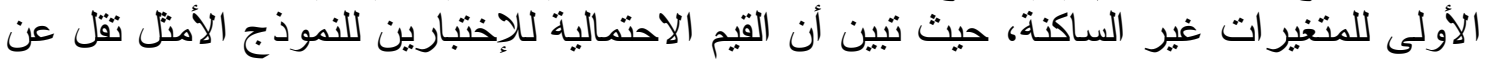

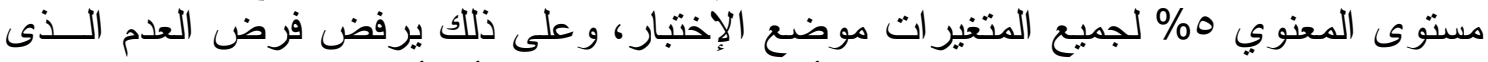

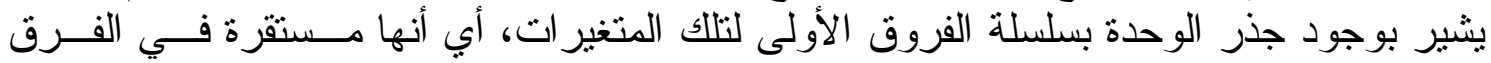

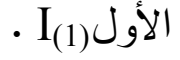

جدول ا. يبين كمية الواردات وسعر الإستيراد للانواع المختلفة من المبيدات الزراعية خلا الفتـرة

( كمية الواردات بالطن، سعر الإستيراد بالألف جنيه للطن)

\begin{tabular}{|c|c|c|c|c|c|c|}
\hline \multicolumn{2}{|c|}{ مبيدات الحشائش } & \multicolumn{2}{|c|}{ المبيدات الفطرية } & \multicolumn{2}{|c|}{ المبيدات الحشرية } & \multirow{2}{*}{ السنو ات } \\
\hline سعر الإستير اد & كمية الوارد ات & سعر الإستير اد & كمية الواردات & سعر الإستير اد & كمية الو ارد ات & \\
\hline$\leqslant, 90$ & 1, & $\wedge, \vee \leqslant$ & $r, 7)$ & 11,0 & $r, 01$ & $r \ldots$ \\
\hline $1 \cdot, 7 V$ & $1, \Gamma \varepsilon$ & IT, ro & Y,OY & $10,7 \leqslant$ & $r, \varepsilon Y$ & $r \ldots 1$ \\
\hline$r \varepsilon, q 1$ & $\cdot, \vee \wedge$ & IV,Tr & Y,Y & $1 \leqslant, 7$. & $r, \wedge T$ & $r \ldots r$ \\
\hline IT,YO & 1,11 & ro,vT & $\cdot, \wedge 9$ & $\{7, Y)$ & r,r & $r \cdot r$ \\
\hline $7, \vee 1$ & T,Y. & 19,79 & T,17 & TY,OS & $\varepsilon, r_{q}$ & $r \ldots \varepsilon$ \\
\hline r.,70 & $\cdot, \wedge \wedge$ & $r q, 1 r$ & 1,11 & rч,q1 & $\Gamma, \leqslant \Lambda$ & r.. O \\
\hline rוT & $1, \wedge$. & $\varepsilon \cdot, \wedge \wedge$ & r, & rA, Ir & $\varepsilon, 9$. & $r \ldots T$ \\
\hline MI,V. & 1,87 & 01,11 & Y,OV & $\leqslant \Lambda, 0$. & $\varepsilon, \vee \wedge$ & $r \cdot \cdot V$ \\
\hline$\varepsilon 1, T V$ & $r, 1$. & rq,9r & $\Gamma, \wedge$. & 01,11 & r, & $r . \lambda$ \\
\hline$Y V, \Sigma V$ & Y,OE & $\varepsilon 1, Y \varepsilon$ & $r, I V$ & ᄀ•,А & r. & $r .99$ \\
\hline $10,9 r$ & r, AO & $\Sigma \neg, V$. & ס ז, & 00,91 & $\varepsilon, r^{\prime}$ & $r \cdot 1$. \\
\hline$\varepsilon r, V T$ & $r, \leqslant \Lambda$ & OY,VY & $0, r$ & $7 \leqslant, 7$. & 0,10 & 4.11 \\
\hline OM, & $r, \wedge I$ & $\sum Y, V T$ & $7, \leqslant \Lambda$ & $V \cdot, V \cdot$ & $\varepsilon, \wedge I$ & $r .1 T$ \\
\hline$\varepsilon \Lambda, r \vee$ & $r, \leqslant q$ & $\varepsilon 9, .7$ & $0,9 \leq$ & $70, \leqslant 7$ & $\varepsilon, Y Y$ & $r .1 T$ \\
\hline 71,71 & T,YV & $0 \wedge, \cdots$ & 0,91 & VT, & $r, 11$ & T. IE \\
\hline $7 \wedge, 7)$ & r, ₹. & $O \wedge, \wedge T$ & $\Lambda, \Gamma \leqslant$ & $7 \cdot, V V$ & $0,0$. & 1.10 \\
\hline$v \cdot, 1 \wedge$ & $\varepsilon, \vee 0$ & $v 1,0$. & $\Lambda, \vee 0$ & $1 \cdots, 0 \mathrm{~V}$ & $\varepsilon, 0$ & $r .17$ \\
\hline IYV,rY & r,r & $1 T \cdot, 11$ & $0, \wedge 9$ & $1 \wedge 9, \wedge 0$ & $r, \leq \varepsilon$ & $r .1 V$ \\
\hline$\varepsilon r, q \mu$ & r.r. & س & $\varepsilon, 1 V$ & 07,19 & r,97 & المتوسط \\
\hline
\end{tabular}

المصدر: جمعت وحسبت : وزارة الزر اعة وأستصلاح الأر اضى،قطاع الشئون الإقتصادية ، نشرة مستلزمات الإنتاج الزر اعى ، أعداد متفرقة. 


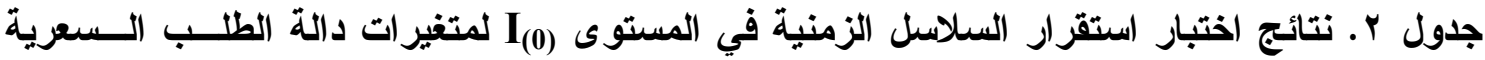

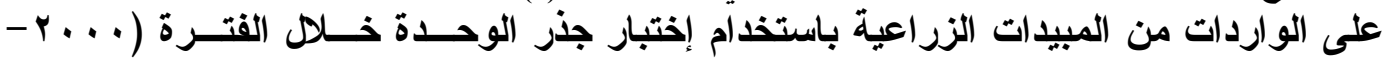

\begin{tabular}{|c|c|c|c|c|c|c|c|}
\hline \multirow[b]{2}{*}{ القـــــار } & \multicolumn{3}{|c|}{$\mathbf{P}_{\mathbf{P}}$} & \multicolumn{3}{|c|}{ ADF } & \multirow{2}{*}{ الإلغتيار } \\
\hline & واتجاه عابت & ثابت فقط & ثابت واتجاه & واتجاه ثابت & ثابت فتط & ثُابت واتجاه & \\
\hline العدم و السلسلة فرض & & $\begin{array}{l}*-r, r . \\
(\cdot, \cdot r)\end{array}$ & $\begin{array}{l}r, \varepsilon)- \\
(\cdot, \cdot \wedge)\end{array}$ & & $\begin{array}{l}* r, r \cdot- \\
(\cdot, \cdot r)\end{array}$ & $\begin{array}{l}r, \varepsilon q- \\
(\cdot, \cdot \vee v)\end{array}$ & الواردات من \\
\hline قبول فرض العدم & & & $\begin{array}{l}\text { *r, }, 9 \wedge- \\
(\cdot, 17)\end{array}$ & & & $\begin{array}{c}*, 1.0- \\
(\cdot, 1 T)\end{array}$ & الواردات من \\
\hline العدم و السلسلة فرض & & & $\begin{array}{l}* 1 \cdot, \wedge \leq- \\
(\cdot, \cdots)\end{array}$ & & & $\begin{array}{l}* 0, r q- \\
(\cdot, \cdots)\end{array}$ & الواردات من مبيدات \\
\hline قبول فرض العدم & & & $\begin{array}{l}* r, O V- \\
(\cdot, \cdot T)\end{array}$ & & & $\begin{array}{l}* r, 70- \\
(\cdot, \cdot 7)\end{array}$ & 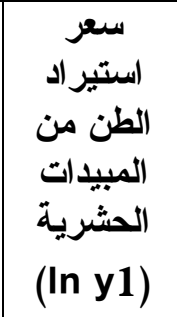 \\
\hline قبول فرض العدم & & & $\begin{array}{l}* r, 70- \\
(\cdot, \cdot T)\end{array}$ & & & $\begin{array}{l}* r, \leqslant q- \\
(\cdot, \cdot \vee \vee)\end{array}$ & الطنتير اد ادن الفبيدات \\
\hline العدم و السلسلة فرض & & & $\begin{array}{l}*\urcorner, \cdot\urcorner- \\
(\cdot, \cdots \wedge)\end{array}$ & & & $\begin{array}{l}* \varepsilon, 77- \\
(\cdot, \cdots 9)\end{array}$ & الطن من منير اد من \\
\hline
\end{tabular}

())الأرقام بين الأقو اس تدل على قيمة الإحتمال prob ، (") تدل على النموذج الأمثل وفقاً لمعنوية الإتجـاه العـام والمصدر: حسبت من بيانات الجدول رقم (1). 


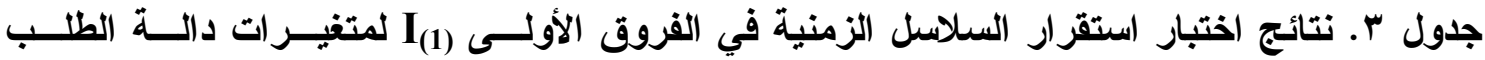

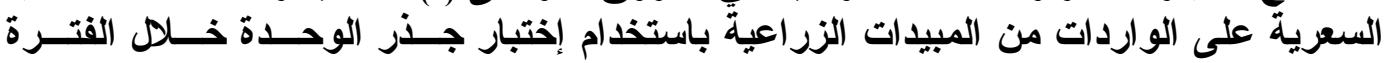

\begin{tabular}{|c|c|c|c|c|c|c|c|}
\hline \multirow{2}{*}{ القــرار } & \multicolumn{3}{|c|}{$\mathbf{P}_{\mathbf{P}}$} & \multicolumn{3}{|c|}{ ADF } & \multirow[t]{2}{*}{ الإختبار } \\
\hline & باتون ثابت & ثابت فقط & $\begin{array}{c}\text { ثابت واتجاه } \\
\text { عام }\end{array}$ & باتجاه ثابت عام & ثابت فقط & 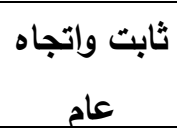 & \\
\hline وفض فرض & $\begin{array}{l}* \varepsilon . \leqslant 0- \\
(\cdots \cdots, r)\end{array}$ & $\begin{array}{l}\varepsilon .7 r- \\
(\cdots \cdot r)\end{array}$ & $\begin{array}{l}\varepsilon .+q- \\
(\cdot .+1)\end{array}$ & $\begin{array}{l}* \varepsilon . \leqslant 0- \\
(\cdots, \cdot r)\end{array}$ & $\begin{array}{l}\varepsilon . \diamond V- \\
(\cdots r)\end{array}$ & $\begin{array}{l}\varepsilon . r Y- \\
(\cdot ., \Lambda)\end{array}$ & كمية الواردات من \\
\hline وفض فرض & - & $\begin{array}{l}* \wedge .79- \\
(\cdots \cdots)\end{array}$ & $\begin{array}{l}v . v \leq- \\
(\cdot \ldots \cdot)\end{array}$ & - & $\begin{array}{l}* \varepsilon .77- \\
(\cdot . \cdot Y)\end{array}$ & $\begin{array}{l}\bullet . r \wedge- \\
(\cdot . \cdot r)\end{array}$ & $\begin{array}{c}\text { سعر استيراد الطن من } 2 \text { المبيدات الحشرية } \\
\text { (In y1) }\end{array}$ \\
\hline وفض فرض & - & $\begin{array}{l}* \vee . \vee ०- \\
(\cdots \cdot \cdot)\end{array}$ & $\begin{array}{l}V . r V- \\
(\cdot \cdots \cdot 1)\end{array}$ & - & $\begin{array}{l}* 0.17- \\
(\cdot .11)\end{array}$ & $\begin{array}{l}\varepsilon . r \Delta- \\
(\cdot . r)\end{array}$ & $\begin{array}{c}\text { سعر استيراد الطن من } \\
\text { المبيدات الفطرية } \\
\text { (Iny2) }\end{array}$ \\
\hline
\end{tabular}

() (الأرقام بين الأقواس تدل على قيمة الإحتمال prob ، (") تدل على النموذج الأمتل وفقاً لمعنوية الإتجاه العام والثابت

المصدر: حسبت من بيانات الجدول رقم (1).

ثانياً: تقدير دوال الطلب السعرية على الواردات من المبيدات الزراعية خلا الفتــرة ( .... ـ-

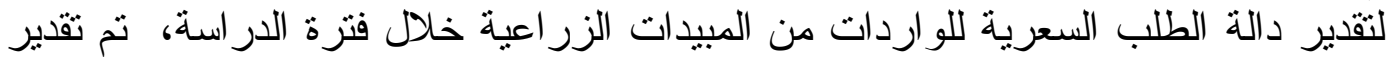

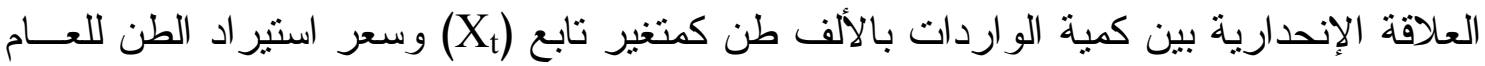

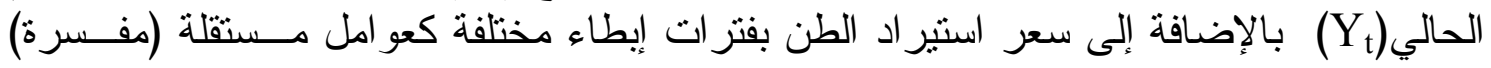

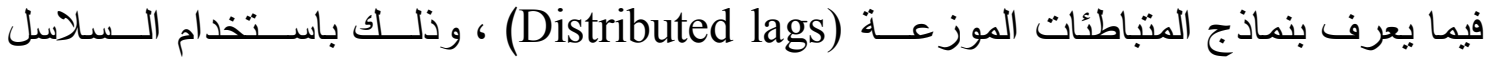

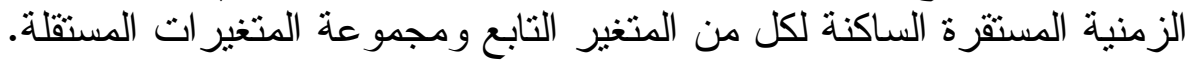

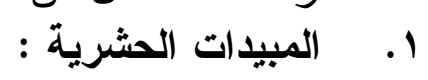

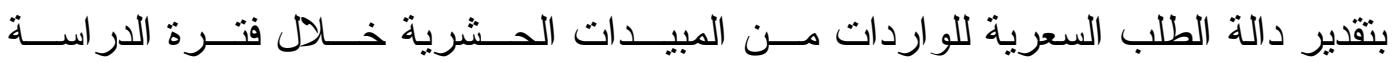

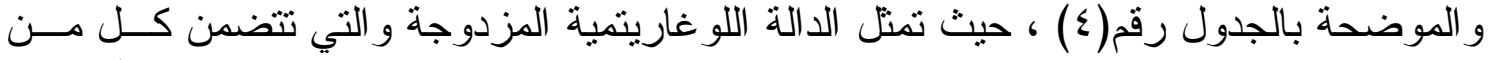

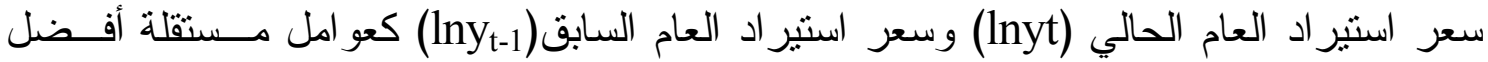

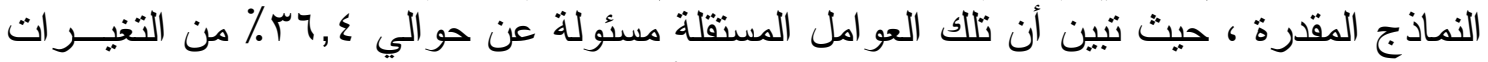

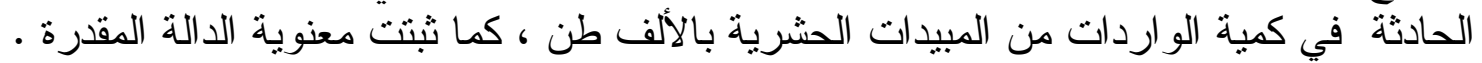

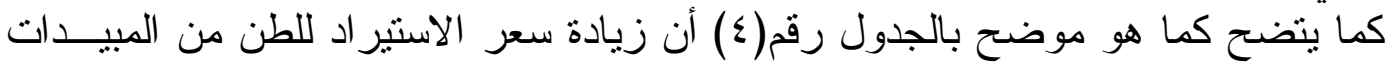

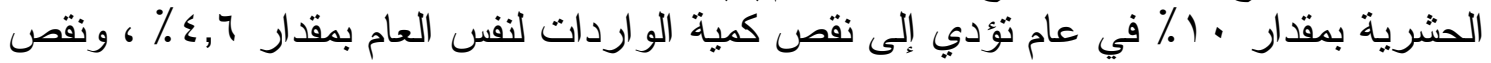

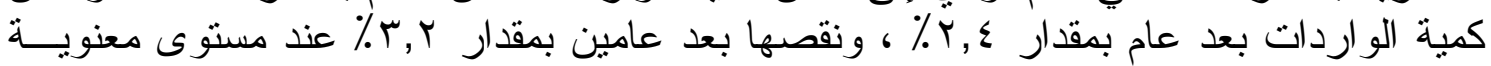




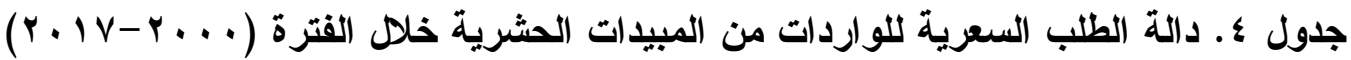

\begin{tabular}{|c|c|c|c|}
\hline Prob` الإحتمال & t t المحسوية & معامل الإنحدار & المتغير \\
\hline$\cdots+$ & r..9- & $\cdot . \leqslant 7-$ & سعر استيراد العام الحالى (Dlnyt) \\
\hline$\cdots r$ & T.M. & $. r \leqslant-$ & سعر استيراد العام السابق (Dlnyt-1) \\
\hline$\cdots 1$ & $1.1 \cdot-$ & $\cdot r r_{-}$ & سعر استيراد العام قبل السابق (Dlnyt-2) \\
\hline$\cdots \cdot$ & Y1.0. &. .77 & الثابت C ب الثاب \\
\hline \multicolumn{4}{|r|}{$($ F-Statistic $)=0.04$} \\
\hline \multicolumn{4}{|c|}{ Prob Of chi square $($ Arch Test $)=0.22$} \\
\hline \multicolumn{4}{|c|}{ Prob of Chi square (L.M test) $=0.58$} \\
\hline \multicolumn{2}{|c|}{ Centered VIF: $\ln y_{t}=1.6$} & $\operatorname{lny}_{\mathrm{t}-1}=2.2$ & $\ln y_{t-2}=1.84$ \\
\hline
\end{tabular}

المصدر : حسبت من بيانات الجداول أرقام ( ) (1).

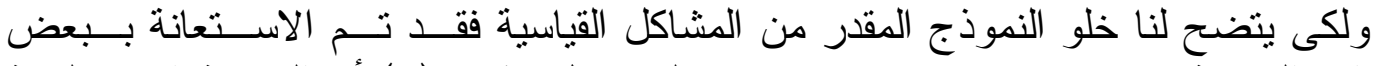

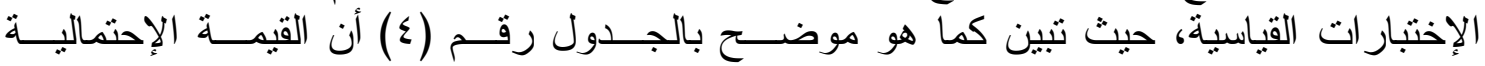

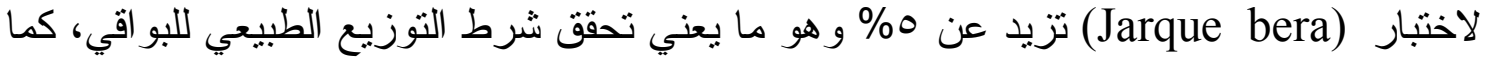

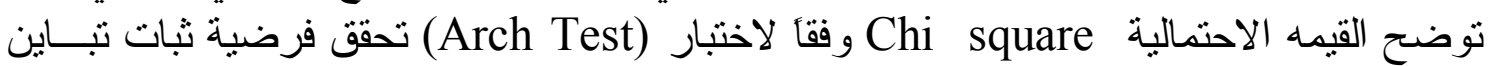

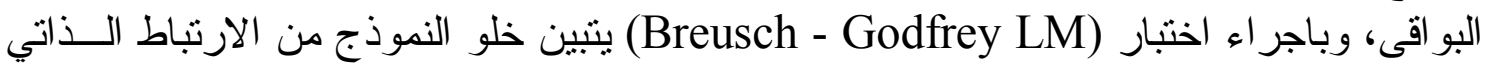

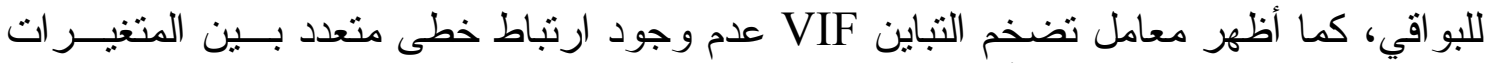
المستقلة موضع الدر اسة حيث أن قيمة المعامل تقترب من الو احد الصد الصحيح.

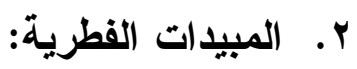

بتقدير دالة الطلب السعرية للواردات من المبيدات الفطرية خلال فترة الدر اسة و الموضحة

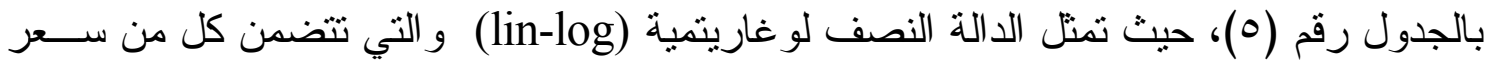

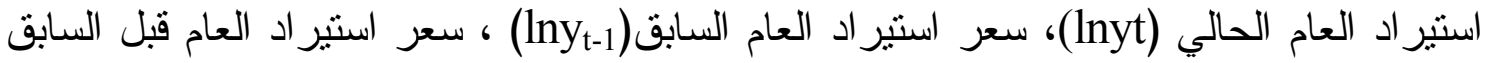
كعو امل مستقلة تؤثز في كمية الواردات من المبيدات الفطرية (lnyt-r)

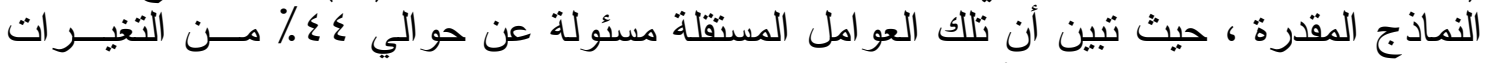

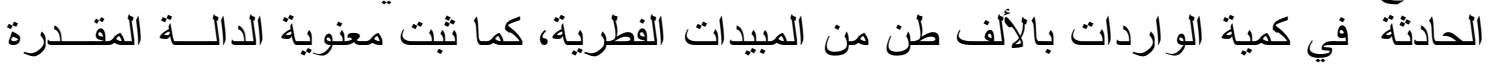

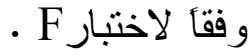

كما يتضح كما هو موضح بالجدول رقم (0)، أن زيادة سعر استير اد الطن من المبيــــات

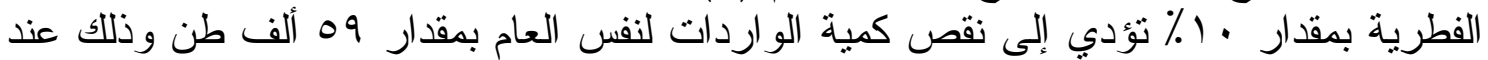

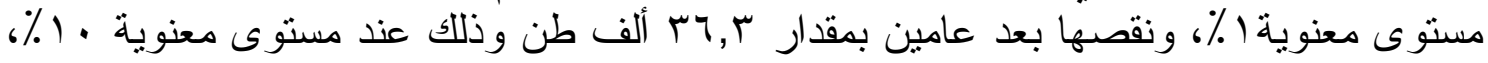

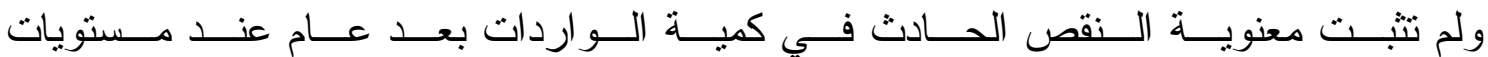
المعنوية (1 \%، 0.6 . 1 \%). 


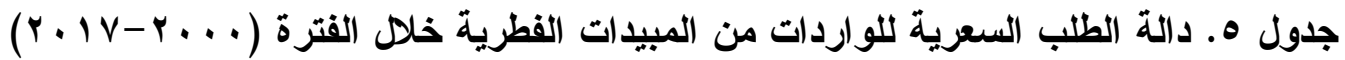

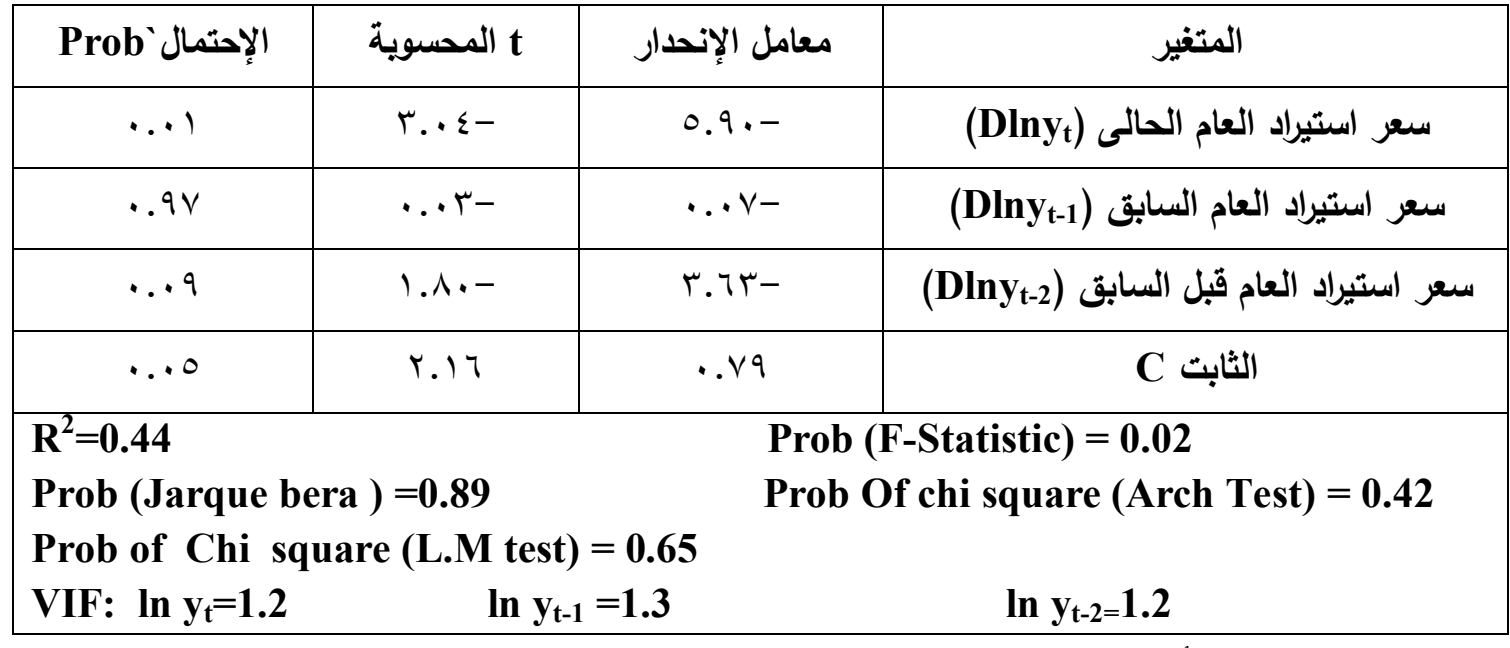

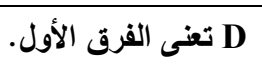

المصدر: حسبت من بيانات الجداول أرقام ( ).

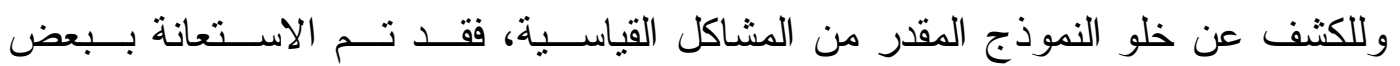

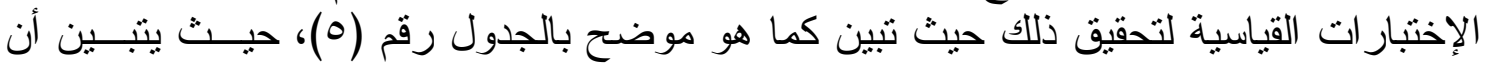

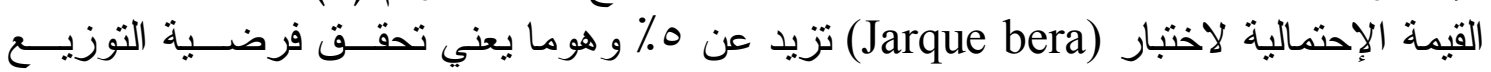

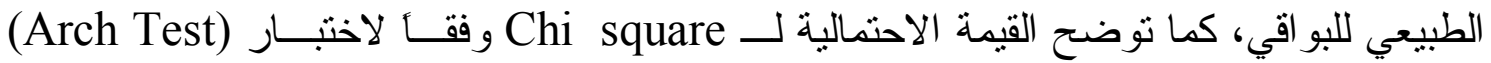
تحقق ثبات التباين، وبإجر اء اختبار (Breusch-Godfrey LM) تبين خلو النموذج من الإرنباط الأذى للبو اقىى. وللكثف عن وجود ارتباط خطي متعدد بين المتغير ات المستقلة موضع الدر اسة، تم تقدير

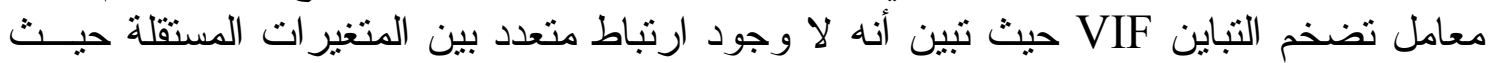
أن قيم VIF تقترب من الو احد الصحيح.

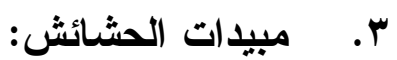

بتقدير دالة الطلب السعرية للواردات من مبيدات الحشائش خلال فترة الدراسة و الموضحة

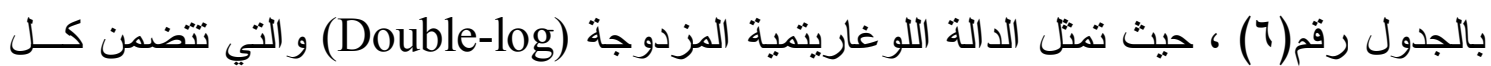

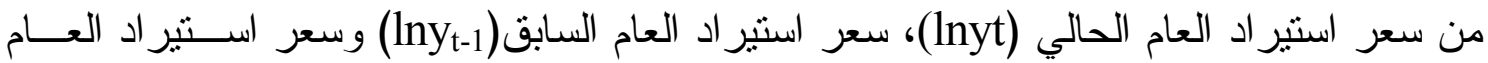

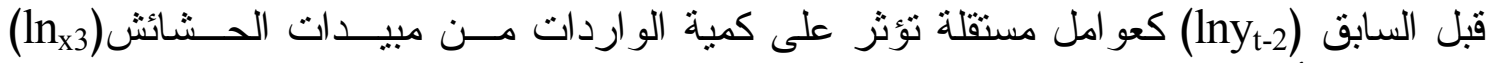

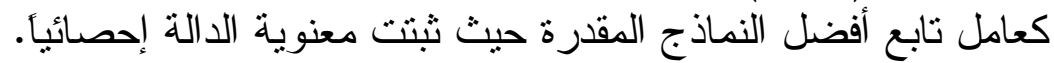

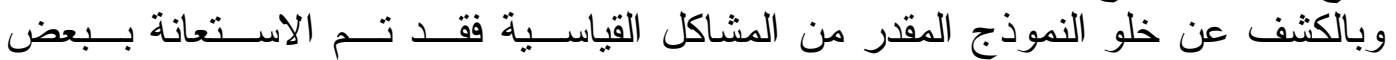

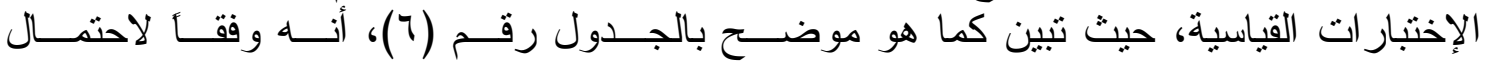

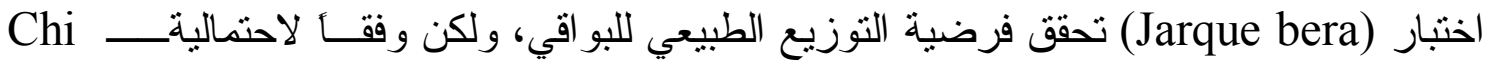

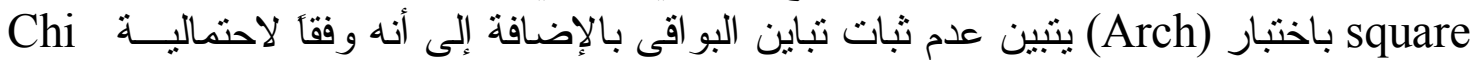

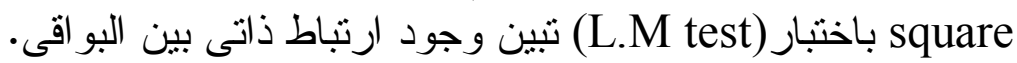

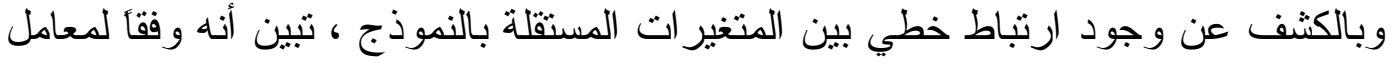
تضخم التباين VIF عدم وجود ارتباط خطى متعدد بين المتغير ات المستقلة موضع الدر استة 


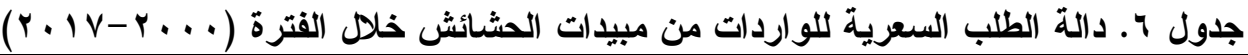

\begin{tabular}{|c|c|c|c|}
\hline Prob` الإحتمال & t t المحسوية & معامل الإنعدار & المتغير \\
\hline .70 & $\because \leqslant 0-$ & $\cdot \ldots \leqslant-$ & سعر استيراد العام الحالى (lnyt) \\
\hline$\cdots+1$ & r...- &. ro- & سعر استيراد العام السابق (lnyt-1) \\
\hline$\cdots 7$ & $r . r_{-}$ & $. M r-$ & سعر استيراد العام قبل السابق (lnyt-2) \\
\hline$\cdots r$ & r.9- &. .09 & الثابت C \\
\hline \multicolumn{3}{|c|}{$\begin{array}{l}R^{2}=0.77 \\
\text { Prob }(\text { Jarque bera })=0.53 \\
\text { Prob of Chi square }(\text { L.M test })=0.08 \\
\text { VIF: } \ln y_{t}=1.65 \quad \ln y_{t-1}=1.89\end{array}$} & $\begin{array}{l}\text { atistic })=0.000 \\
\text { i square }(\text { Arch } \text { Test })=0.07\end{array}$ \\
\hline
\end{tabular}

المصدر: حسبت من بيانات الجداول أرقام ( ).

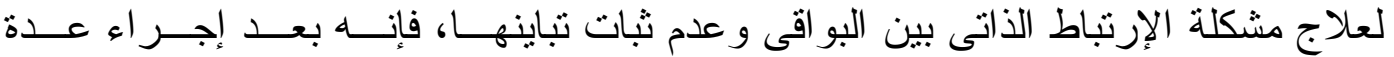

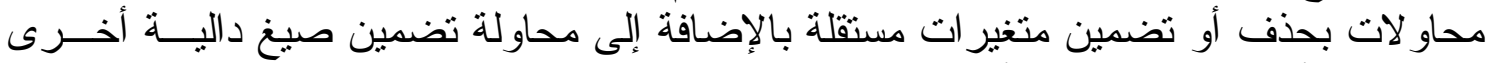

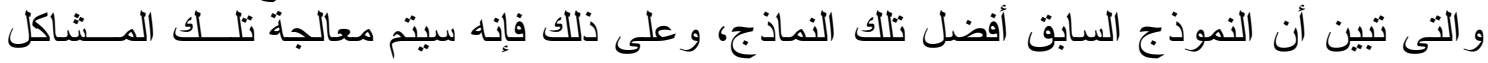

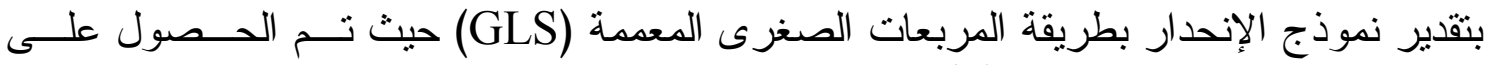

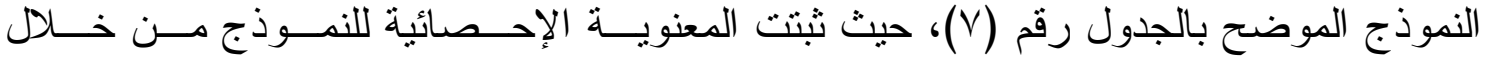
إحصائية Fo

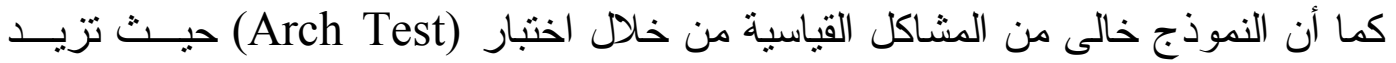

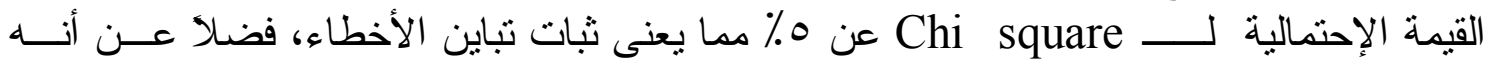

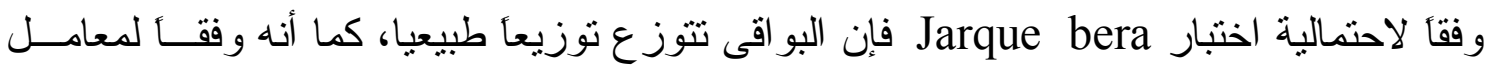
تضخم التباين VIF فإنه لا يوجد ارتباط متعدد بين المتغير ات المستقلة

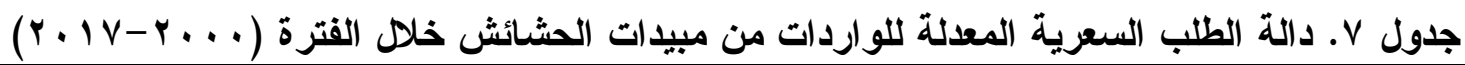

\begin{tabular}{|c|c|c|c|}
\hline Prob` الإحتمال & t t t t & معامل الإنحدار & 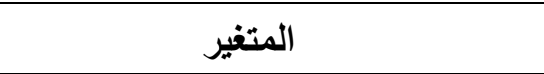 \\
\hline$\cdots r$ & r.or- & $\cdot Y_{Y-}$ & سعر استيراد العام الحالى (lnyt) \\
\hline$\cdots \varepsilon$ & r.r.- & $\cdot r \leqslant-$ & سعر استيراد العام السابق (lnyt-1) \\
\hline$\cdots \wedge$ & $1 . \wedge 7-$ &.$Y_{T-}$ & سعر استيراد العام قبل السابق (lnyt-2) \\
\hline.$Y_{1}$ & $1 . r 1$ &..$\leqslant r$ & AR(1) \\
\hline $\begin{array}{l}\mathrm{R}^{2}=0.53 \\
\text { Prob (Jarque } \\
\text { D.W }=2.14 \\
\text { VIF: } \ln y_{t}=9.2\end{array}$ & $=0.53$ & $\begin{array}{c}\text { Prob } \\
\text { Prob }\end{array}$ & $\begin{array}{l}\text { atistic })=0.001 \\
\text { i square }(\text { Arch Test })=0.42 \\
t-2=9.9\end{array}$ \\
\hline
\end{tabular}

المصدر : حسبت من بيانات الجداول أرقام ( ) (1)

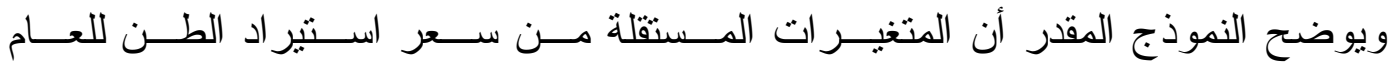

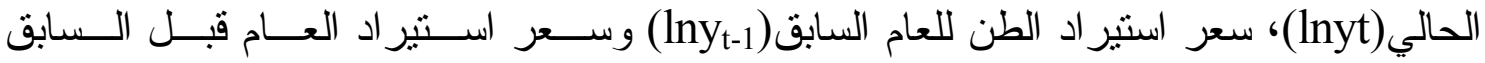
(lnyt-2)

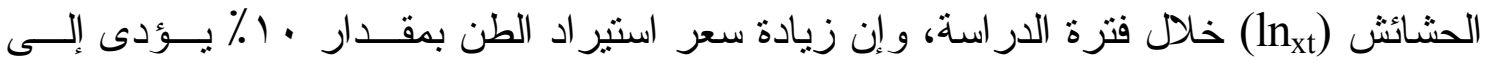

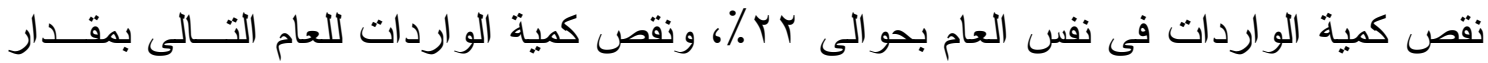




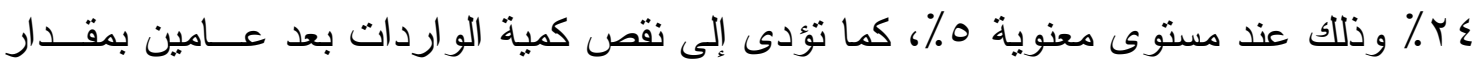

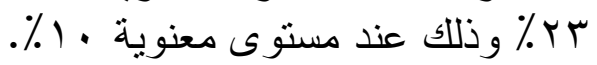

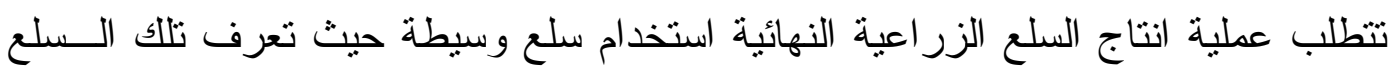

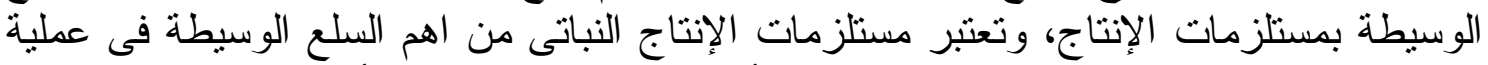

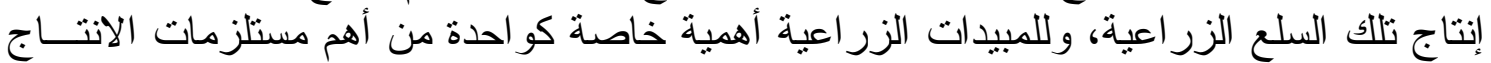

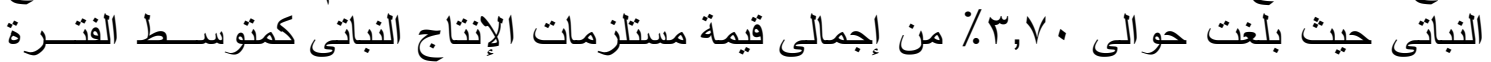

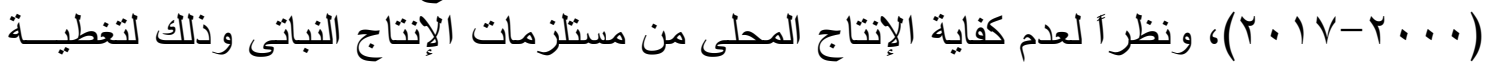

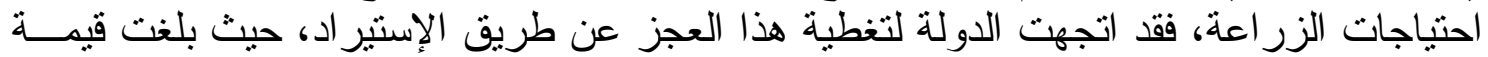

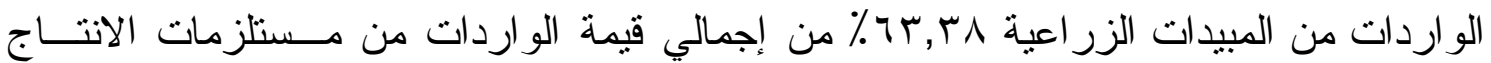

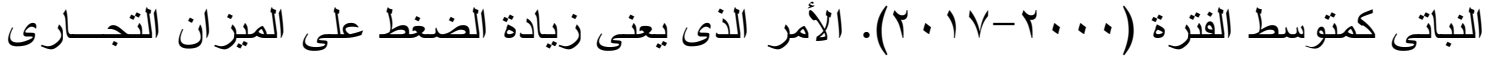

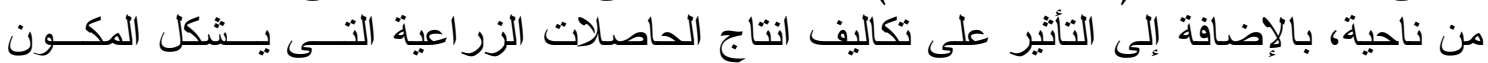

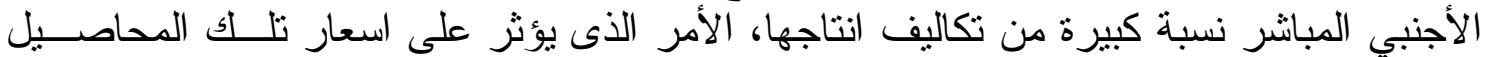

و المساحات المزرو عة منها.

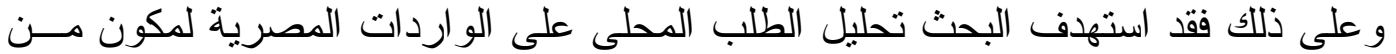

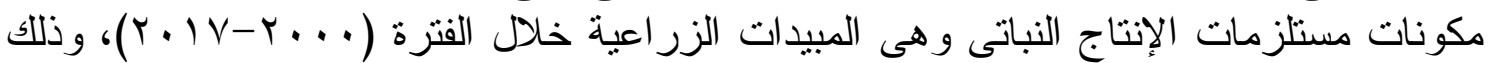

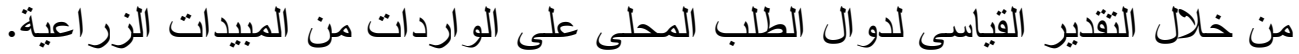

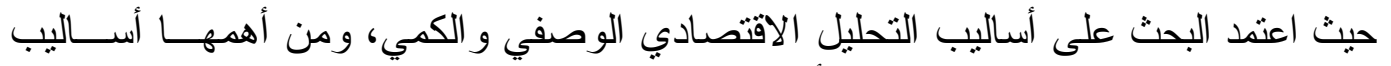

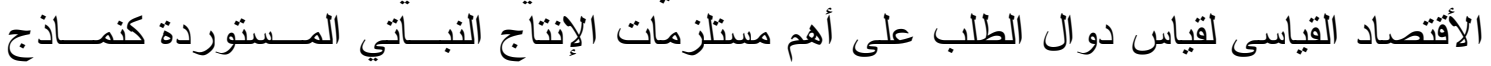

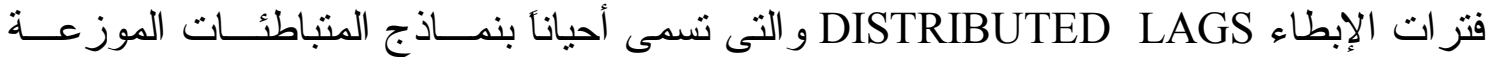

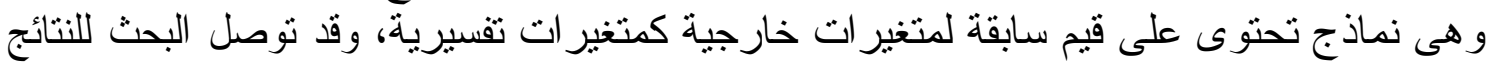

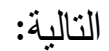

1 - فيما يتعلق بالواردات من المبيدات الحشرية: بتقدير دالة الطلب السعرية للــواردات

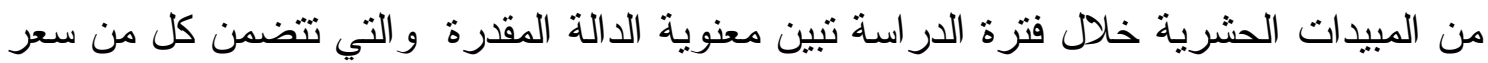

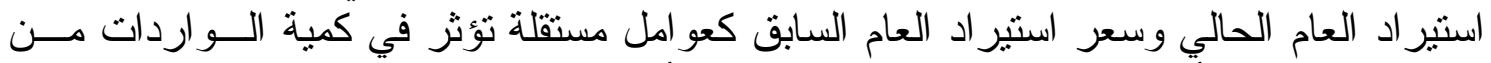

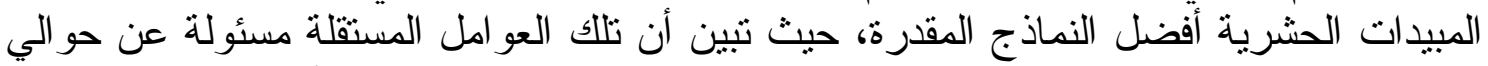

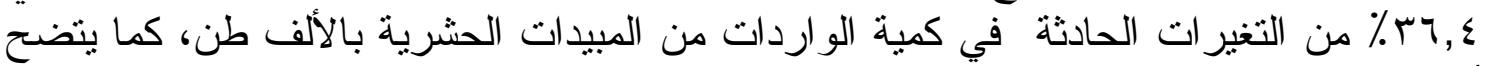

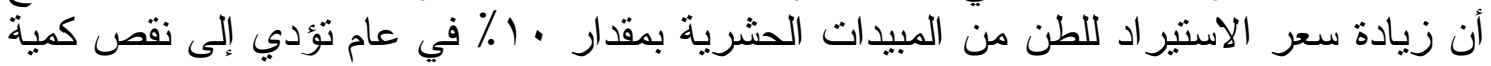

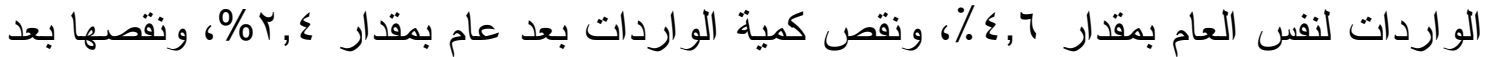

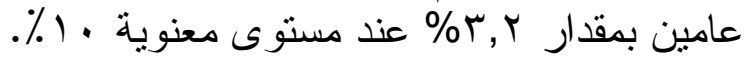

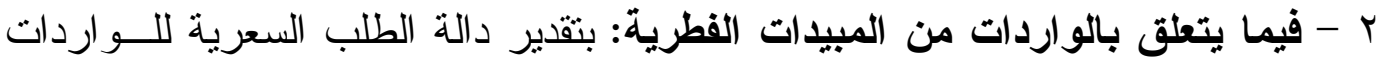

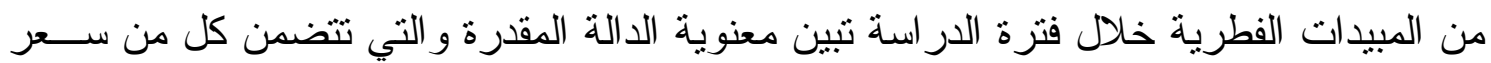

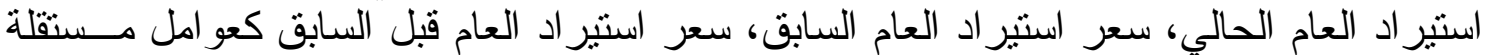

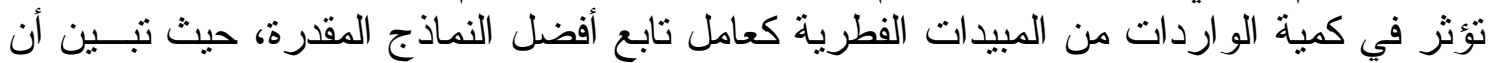

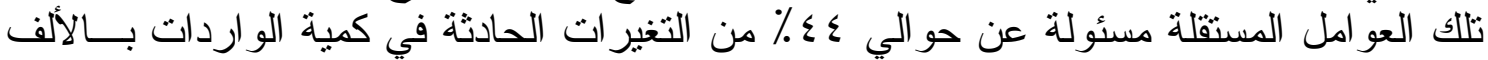

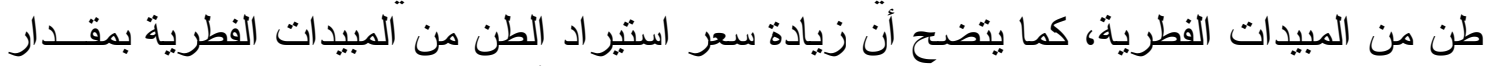

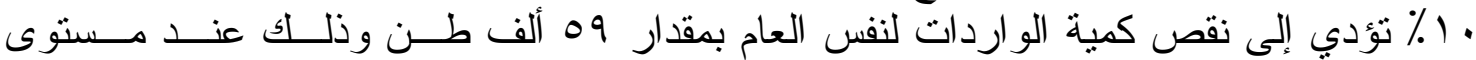

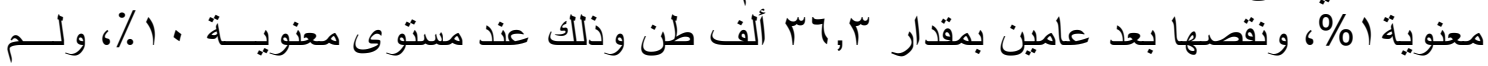

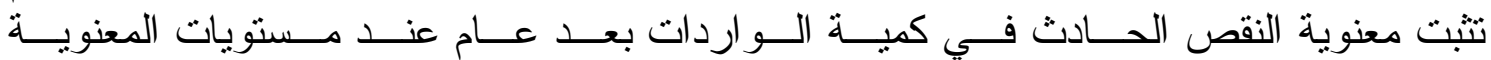
. $(\%)$. $6 \% 06 \%)$ 
r - فيما يتعلق بالواردات من مبيدات الحشائش: بتقدير دالة الطلب السعرية للــواردات

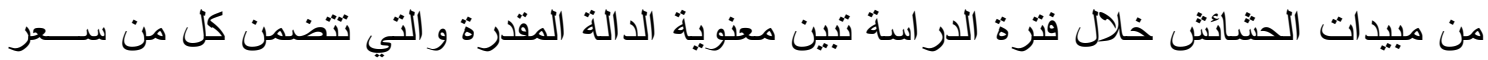

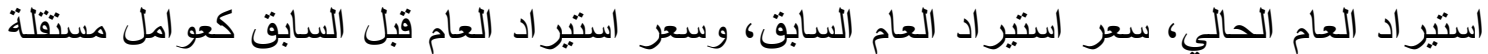

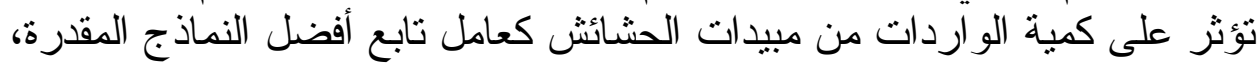

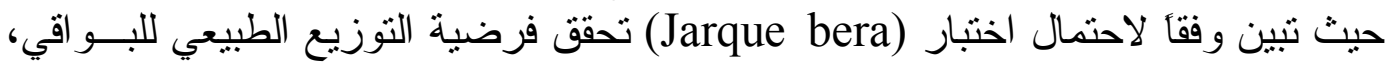

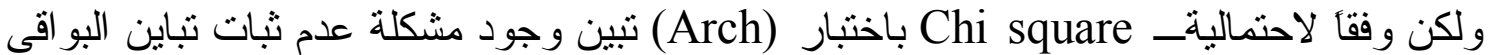

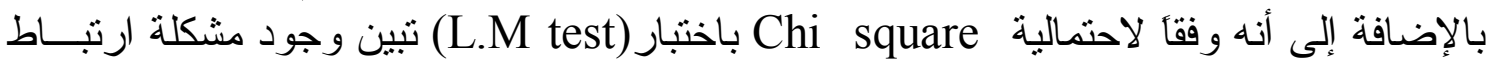

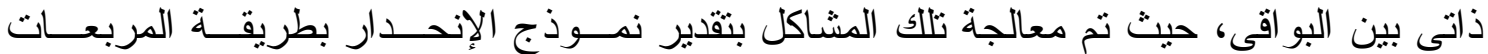

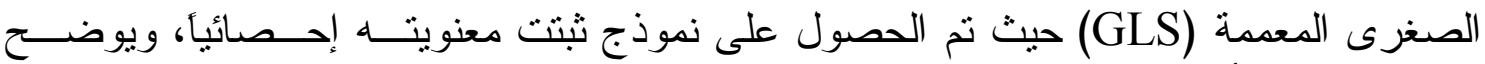

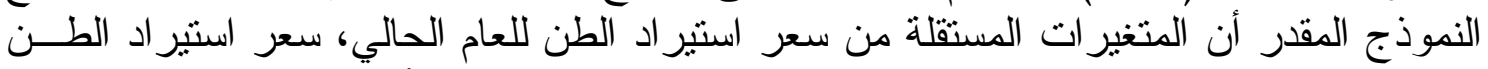

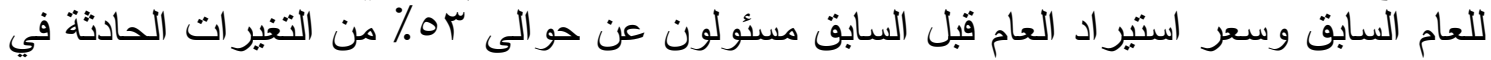

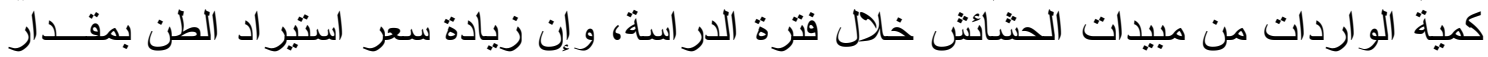

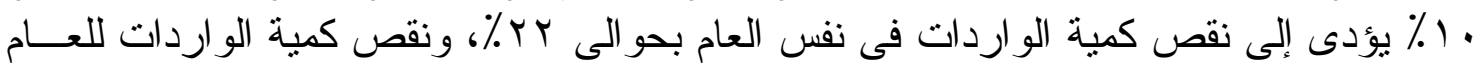

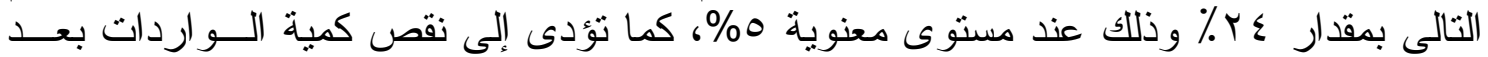

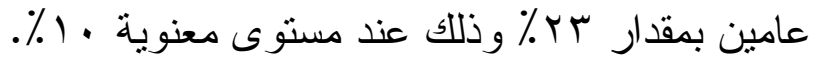

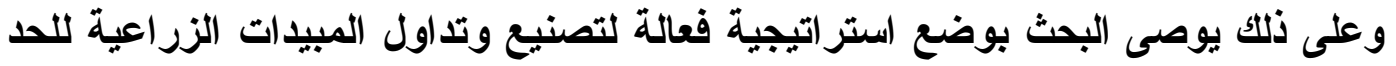

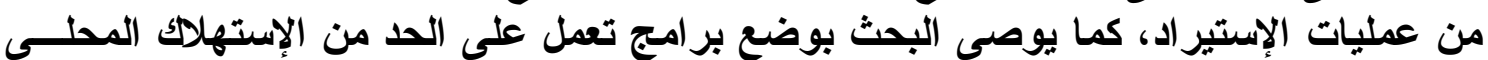

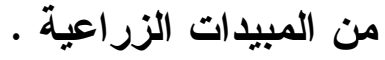

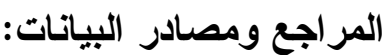

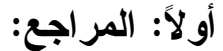

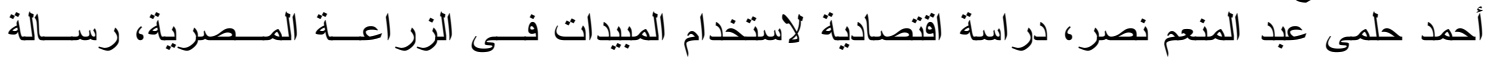

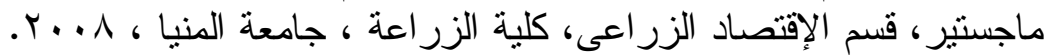

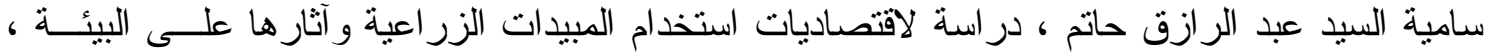

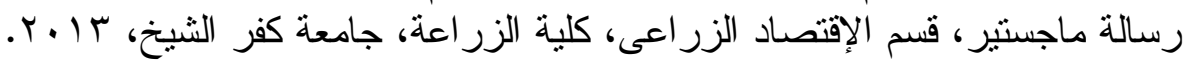

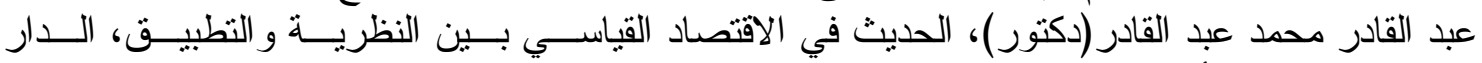

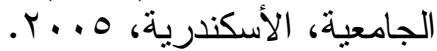

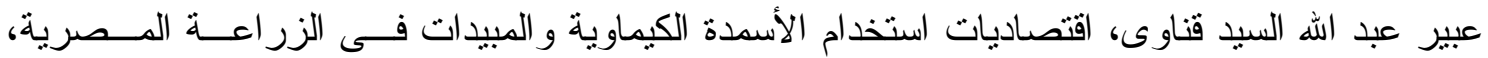

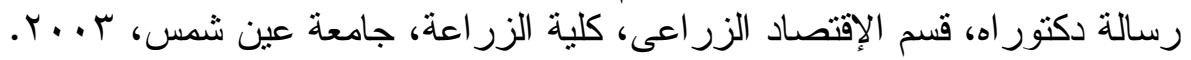

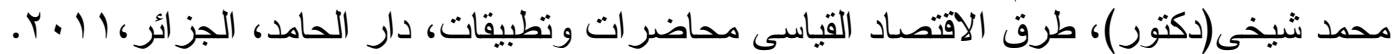

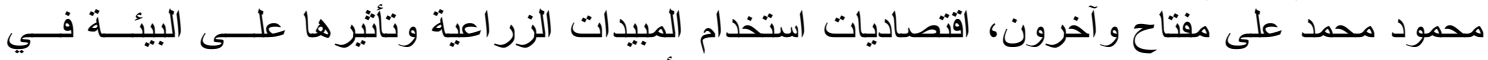

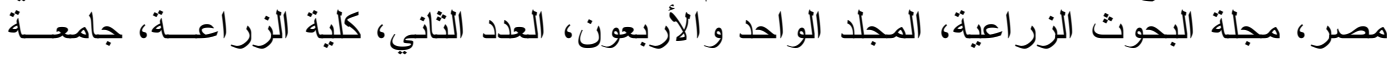

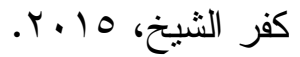

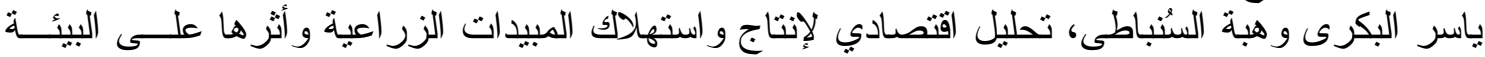

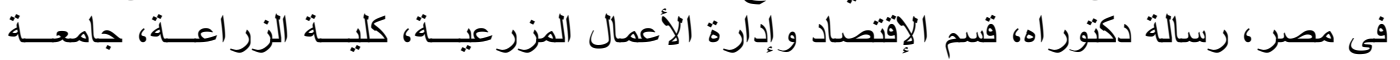

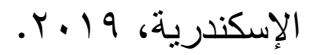

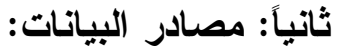
الجهاز المركزى للتعبئة العامة والإحصاء، النشرة السنوية لتقديرات الدخل من القطاع الزراعى، أعداد

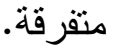

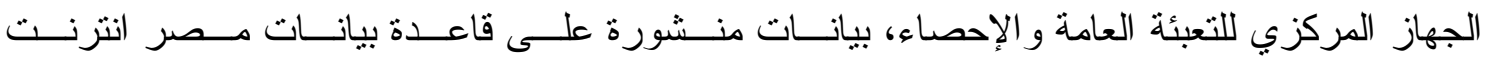
.www.Capmas.org.eg وزارة الزراعة واستصلاح الأراضي، قطاع الثئون الاقتصادية، نشرة مستلزمات الإنتاج الزراعسى،

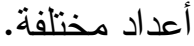




\section{An Economic Study of the Import Demand Functions of Agricultural} Pesticides

\section{Dalia Hamed EI Showeikh; Yaser Abdel-Hamed Diab; Ramy Ahmed Abdel-Hafez and Hamdy Omar Zarea Hassan}

Department of Agricultural Economics, Faculty of Agriculture, Assiut University, Assiut, Egypt.

\section{Summary}

The research aimed to analyze the domestic demand for Egyptian imports of a component of plant production supplies, namely agricultural pesticides during the period (2000-2017), through the measurement of the level of domestic demand for imports of agricultural pesticides.

The research was based on methods of descriptive and quantitative economic analysis, the most important of which are the methods of the economic measurement to measure the demand for the most important supplies of plant production imported such as models of slowing periods (DISTRIBUTED LAGS), and the research has reached the following results:

1- With regard to imports of pesticides: the price demand function of pesticide imports during the study period is estimated to show the morale of the estimated function, which includes both the current year's import price and the previous year's estered price as independent factors affecting the quantity of imports from Pesticides are the best models estimated, as these independent agents were found to be responsible for about $36.4 \%$ of the changes in the amount of imports of pesticides in 1,000 tons, and it is clear that the increase in the price of imports per ton of pesticides by $10 \%$ In a year leading to a decrease in the volume of imports for the same year by $4.6 \%$, decrease in the amount of imports after a year by $2.4 \%$, and decreased after two years by $3.2 \%$ at a level of $10 \%$.

2- The price demand function of imports of fungicides is estimated during the study period to show the morality of the estimated function, which includes both the price of the current year's import, the price of the previous year's import, the price of the previous year's import, the price of the previous year's importation as independent factors affecting In the quantity of imports of fungicides as a dependent factor of the best estimated models, where it was found that these independent factors are responsible for about $44 \%$ of the changes occurred in the amount of imports in 1,000 tons of fungicides, and it is also clear that the increase in the price of the import of a ton $10 \%$ of fungicides lead to a reduction in the amount of imports for the same year by 59 thousand tons, at a moral level of $1 \%$, and a decrease after two years by 36.3 thousand tons, at a moral level of $10 \%$, and did not prove the meaning of the decrease in the amount of imports after a year at moral levels $(1 \%, 5 \%, 10 \%)$.

3- The price demand function of imports of herbicides is estimated during the study period to show the morale of the estimated function, which includes both the current year's estermed price, the previous year's import price, and the price of imports of the previous year as separate factors affecting the amount of imports of herbicides as a factor of the best estimated models, The estimated 
model shows that the independent variables of the price of import per ton for the current year, the price of the import of a ton for the previous year and the price of imports of the previous year are responsible for about $53 \%$ of the changes in the amount of imports of herbicides during the study period, and that the increase in the price of imports per ton by $10 \%$ leads to a decrease in the amount of imports in the same year by about $22 \%$, and the decrease in the amount of imports for the following year by $24 \%$, at a moral level of $5 \%$, and lead to a decrease in the quantity after two years by $23 \%$ and that At a moral level of $10 \%$.

Therefore, research recommends the development of an effective strategy for the manufacture and circulation of agricultural pesticides to reduce imports, and it is recommended to develop programs to reduce the local consumption of agricultural pesticides. 